\title{
Regrasp Planning Considering Bipedal Stability Constraints
}

\author{
Daniel Sánchez ${ }^{1}$, Weiwei Wan ${ }^{1,2, *}$, Kensuke Harada ${ }^{1,2}$, Fumio Kanehiro ${ }^{2}$
}

\begin{abstract}
This paper presents a Center of Mass (CoM) based manipulation and regrasp planner that implements stability constraints to preserve the robot balance. The planner provides a graph of IK-feasible, collision-free and stable motion sequences, constructed using an energy based motion planning algorithm. It assures that the assembly motions are stable and prevent the robot from falling while performing dexterous tasks in different situations. Furthermore, the constraints are also used to perform an RRT-inspired task-related stability estimation in several simulations. The estimation can be used to select between single-arm and dual-arm regrasping configurations to achieve more stability and robustness for a given manipulation task. To validate the planner and the task-related stability estimations, several tests are performed in simulations and real-world experiments involving the HRP5P humanoid robot, the 5th generation of the HRP robot family. The experiment results suggest that the planner and the task-related stability estimation provide robust behavior for the humanoid robot while performing regrasp tasks.
\end{abstract}

\section{INTRODUCTION}

In this paper, we present regrasp planner considering the balances of humanoid robots. It eliminates unstable poses for the robots during manipulation, and enables the robot to perform dexterous tasks with one or two arms, avoiding collisions and falling. Both simulations and real-world tests are performed to validate the utility of this solution. Furthermore, the planner is used to compare different robot stances for complicated regrasp tasks.

Regrasp planning involves several important considerations such as the initial and goal poses of a manipulated object, the different grasping poses available to the robot in order to handle the object, and the intermediate poses of the robot for achieving the object's goal pose. Further considerations and constraints are also implemented so the regrasp task can be performed correctly, such as collision constraints that forbid the robot from performing movements that may make it collide with the environment or itself. One often overlooked consideration for the successful regrasp planning and manipulation of an object is the balance of the robot-object system, which proves to be especially important for unfixed robotic platforms such as humanoid robots and wheeled robot. These robots might fall out of balance while performing these operations with heavy or asymmetrical objects.

To create a planner that considers the robot balance, this paper includes CoM-based constraints in a regrasp planner to enhance the robustness and stability of the robot poses for the robot-object system. By taking into account the CoM of the

${ }^{1}$ Graduate School of Engineering Science, Osaka University, Japan. ${ }^{2}$ National Inst. of AIST. *Correspondance author: Weiwei Wan wan@hlab.sys.es.osaka-u.ac.jp system and selecting a minimum threshold distance between the robot-object system CoM and its support polygon, unstable poses are discarded to preserve the balance of the robot. The CoM constraints can be also used for choosing a robot stance that maximizes the amount of stable and collision-free poses for the regrasp task. The use of this CoM based planner is useful for humanoid manipulation under a fixed-leg stance such as the robot standing on one leg.

The CoM constraints in our regrasp algorithms for singlearm and dual-arm regrasp [1] [2] were tested by performing several simulations and real-world tests using the HRP5P robot, the 5th generation of HRP humanoid robots [3]. For the simulations, several regrasp tasks were performed in order to compare the change of the robot-object system CoM movement with different thresholds. A random-biased sampling and evaluation of robot states, inspired by RRT [4] is also performed for single-arm and dual-arm regrasping. The results of these experiments provide a means to measure and compare how stable single-arm regrasp and dual-arm regrasp tasks are performed in different circumstances, such as different robot stances or varying object mass and CoM. These comparisons can then be used to select a motion that provides the most stable robot posture. For real-world tests, the planner is implemented in the HRP5P humanoid robot. The robot is tasked to reorient an electric drill: The drill pose and position have to be changed by using dualarm regrasp for which our planner identifies and eliminates several unstable poses. The robot is able to successfully complete the given tasks while avoiding poses that place its CoM too close to the edge of the robot support polygon, assuring a robust motion.

\section{Related Work ANd Contributions}

For several years, regrasp planning has been the object of attention for different studies. When an object's pick-up grasp is incompatible with its put-down grasp, regrasp can be performed to achieve the goal pose [5], which is often the solution for many tasks in robotic applications. Some early work includes the regrasp planning of robotic manipulators such as [5], [6] and [7], but those solutions only considered one-armed robots with a fixed position, therefore they lack specific considerations that must be taken into account for humanoid robots such as HRP3 [8], WALK-MAN [9] and TALOS [10].

Recently, several manipulation and regrasp planners have been proposed for dual-armed robots with a focus on efficient and collision free object manipulation. In [2], comparison of different algorithms for single-arm and dual-arm regrasp were performed in different scenarios. In [11] a planner for a robot to keep an object stable under a sequence of 
external forces was proposed. A CoM-based grasp pose adaptation method for picking up objects with one arm was introduced in [12]. It used 3D perception and force/torque feedback to reduce the load of joints during manipulation. In [13], a planner for autonomous in-hand manipulation using finger gaiting was presented. Also, some researchers have worked on manipulation planning on constraints such as in [14]. Some optimization-base motion planning algorithms are summarized in [15]. These work offered several solutions for regrasp planning tasks, but they failed to consider the balance of robot-object system. In this regard, our planner presents a CoM-based solution that implements stability constraints during regrasp planning.

On the other hand, several studies have been done to balance walking robots. In [16], for example, a series of static and dynamic stability criteria were evaluated for different environments. An early work [17] presented the Center of Pressure Method (CPM) and declared that a robot is dynamically stable if the projection of its $\mathrm{CoM}$ along the direction of the resultant force acting on the CoM is inside its support polygon. Since then, more refined methods for posture and stability control have been proposed and studied: In [18] a stability analysis and momentum based control architecture that avoided instabilities at the zero-dynamics level was introduced. In [19] an experimental comparison between a fully model-based control approach and a biologically inspired approach derived from human observations was shown. A whole-body control for balancing and pose stabilization using optimization of contact forces and Model Predictive Control (MPC) was presented in [20]. A stability strategy that used the robot CoM height in its control law was proposed in [21]. In [22], a comprehensive study of Team WPI-CMU's approach to the DARPA Robotics Challenge (DRC), was shown, it focused on the team's strategy to avoid failures and prevent the robot from falling. Finally, in [23] an iterative contact point estimation method for estimating the stability of actively reconfigurable robots was presented.

The aforementioned control methods suggested general solutions for robot walking and static balancing, but they did not address complicated tasks such as object manipulation. For this reason, researchers begin to study stability-based manipulation. In [24] for example, a method was proposed for achieving balance during transporting heavy objects. Also, in [25] a humanoid robot designed for dynamic manipulation was presented. In [26], a preliminary planner that considered the robot CoM during manipulation was proposed, without taking into account object properties. In [27] a strategy for a humanoid robot to pull a fire hose and reach a desired goal position was discussed. These work did not take into account more dexterous tasks such as reorienting objects and handover, which leads to drastic change in the robotobject system CoM. Performing these more dexterous tasks can prove to be dangerous when the mass of the object is relatively high or when the robot is in sub-optimal stances for balance.
Our planner addresses the aforementioned concerns by implementing CoM-based constraints for the robot-object system, assuring that the robot does not incur in unstable poses, increasing the static stability of the system by keeping its CoM within a minimum distance from the boundary of its support polygon.

The CoM constrained planner is an enhanced version of our previous planner [2], it builds a regrasp graph by connecting nodes between given initial and goal placements for an object, and searches the graph to find a sequence of pick-and-place sub-tasks. This generates a sequence of states, grasps, transit and transfer motion, connecting the starting and ending nodes. This paper improves the previous planner by evaluating if the state of the robot-object system is stable, in order to preserve its balance during searching. If the state is IK-unfeasible, generates a collision, or makes the robot fall out of balance, the corresponding node is removed and the search continues until a set of nodes connecting the initial and goal poses is found.

The stability of a given state is computed using the CoM of the robot-object system and the robot support polygon, this provides a means to measure how stable the robotobject system is. By determining the CoM of the system and its projection over the robot support polygon plane, we can determine if the robot is stable [28]. For this work, it is assumed that the robot is standing on an even and flat surface while performing regrasp tasks. This enables the planner to evaluate the states of the robot without extra sensors and complicated calculations.

\section{Stability Measurement and Constraints}

In order to generate stable motion sequences for the robot, the planner determines the global position of the robot-object system CoM for each robot state. Given the CoM global position and the robot feet position, the planner checks if the projection of the CoM is inside the support polygon of the robot and its distance to the polygon edges. If the distance is below a given threshold, the state is discarded and a new graph search is started.

The proposed method can also be used to explore the stability of a task: By randomly checking the stability of the possible robot states for the task completion in a biased fashion, a highly task-related stability measurement can be performed by determining the ratio of stable evaluated robot states and the total amount of evaluated poses.

\section{A. Calculation of robot-object system CoM}

In our simulator, a local coordinate system is defined and used to determine the changes of joints. With the rotation matrices of the robot links, it is possible to obtain the global position of the robot limbs and the manipulated object, as well as their CoMs during regrasp. Thus, we compute the CoM of the whole system, $\Gamma^{S}(x, y, z)$ using

$$
\Gamma^{S}(x, y, z)=\frac{M \Gamma^{\text {robot }}(x, y, z)+m \Gamma^{\text {object }}(x, y, z)}{M+m}
$$


where $M$ and $m$ are the masses of the robot and the object respectively and $\Gamma^{\text {robot }}(x, y, z)$ and $\Gamma^{\text {object }}(x, y, z)$ are their CoMs.

Once the CoM of the system is calculated, the robot support polygon is computed using the convex hull formed by its feet. For a robot pose to be considered stable, the projection of $\Gamma^{S}(x, y, z)$ must be inside the support polygon and the minimum distance between the projection and the edges of the convex hull polygon must be higher than a given threshold. If a robot pose/state does not comply with these constraints, the pose is discarded and a new search is started.

\section{B. Task stability measurement}

Since humanoid robots can perform one-hand or dualarm regrasp, it is of interest to find task-related stability estimations for the different regrasping solutions a humanoid robot may have for a task. The state evaluation is performed using a energy based search method inspired by RRT, which presents a biased search towards low-cost regions such that solution paths remain close to minimal work paths [29].

The biased search can be used to perform a stability measurement highly related to a specific task: by performing a continuous state exploration, several random but goalbiased robot poses can be evaluated and a ratio of stable poses over explored poses can be calculated by using Eqn.(2).

$$
R_{\text {hand }}=\frac{C_{\text {hand }}}{C_{\text {hand }}+U_{\text {hand }}}
$$

where $R_{\text {hand }}$ is the stability ratio between the stable poses $C_{\text {hand }}$ and the total amount of explored poses which also includes the unstable poses $U_{\text {hand }}$. This ratio is calculated for a particular robot leg stance and a given hand configuration hand, the hand configuration indicates which hand is going to be used for the task in the case of a one-armed regrasp task or in which order the hands will be used, in case of dual-arm regrasp. Given the randomness of the RRT exploration and the introduced bias towards the starting and ending points of a given path for performing the task, this method represents a task-related stability estimation, which can be used to choose between single-arm and dual-arm regrasp or between the use of the left or right hand for a given regrasp task, maximizing the robustness and stability of the motions of the robot by using the hand configuration with the highest stability ratio $R_{\text {hand }}$.

\section{Simulations and Real-world Results}

To test the constrained planner, a series of simulations were performed using an HRP5P model. In the simulations, the robot is required to change the pose of a given object and move it to a goal position, as seen in Fig 1 . The HRP5 has 35 DoFs making it a suitable platform for highly complex motions and dexterous manipulation. Four different stances were simulated, in each case, the task stability measurement method was used to determine the stability ratio for the different hand configurations and stances available to the robot. The planner is also tested using a real robot. Two challenging tasks involving object regrasping were given to the robot to test the planner ability to generate a motion path for the robot that complies with the imposed constraints to its CoM location. The results of these tests are discussed in this section, too.

\section{A. Simulations}

1) Upright stance: In this case, the robot assumes an upright posture, with its feet in symmetrical positions. A minimum stability threshold of $60 \mathrm{~mm}$ was given to the regrasp planner. The manipulated object has a mass of 8 $K g$. Its CoM is located at $(150,80,150) \mathrm{mm}$ in the object's local coordinate system. The object has its initial position on the robot right-hand side and it is laying sideways on a table, while the object's goal position is on the robot's left-hand side. The results of task stability measurement are shown in Table I. The changes of CoM in the projection plane are shown by blue curves in Fig 2 In the same figure, a comparison to threshold $0 \mathrm{~mm}$ is shown in red curves.

TABLE I: Upright Stance: Path evaluations

\begin{tabular}{lccc}
\hline Hand configuration & $C_{\text {hand }}$ & $U_{\text {hand }}$ & $R_{\text {hand }}$ \\
\hline Start from left hand - End with left hand & 10529 & 15 & 0.9986 \\
Start from right hand - End with right hand & 15991 & 1801 & 0.8998 \\
Start from left hand - End with right hand & 11189 & 41 & 0.9963 \\
Start from right hand - End with left hand & 5391 & 7351 & 0.4231 \\
\hline Meanings of abbreviations $C_{\text {hand }}$ : \# of stable poses; $U_{\text {hand }}$ : \# of unstable poses; $R_{\text {hand }}$ : \\
Ratio between $C_{\text {hand }}$ and $C_{\text {hand }}+U_{\text {hand }}$; Hand configuration: The robot will \\
plan a motion between two left-hand, right-hand, or left-to-right-hand grasps. \\
If the grasps were from different hands, the robot will plan a handover motion.
\end{tabular}

2) Staggered stance: For this simulations, the robot assumes a staggered posture with its right leg $50 \mathrm{~mm}$ in front of the robot local coordinate system and its left foot 200 $\mathrm{mm}$ behind. In this case, the object has a mass of $5 \mathrm{Kg}$ and the minimum threshold given to the planner was of 30 $\mathrm{mm}$. The object has the same origin and goal poses as the upright stance simulation, but its CoM is located at $(-150,80$, 150) $\mathrm{mm}$ of the object's local coordinate system. The results of task stability measurement are shown in Table II The changes of CoM in the projection plane are shown in blue curves in Fig 3 A comparison with the minimum threshold set to $0 \mathrm{~mm}$ is shown in red curves in the same figure.

TABLE II: Staggered Stance: Path evaluations

\begin{tabular}{lccc}
\hline Hand configuration & $C_{\text {hand }}$ & $U_{\text {hand }}$ & $R_{\text {hand }}$ \\
\hline Start from left hand - End with left hand & 6447 & 135 & 0.9795 \\
Start from right hand - End with right hand & 250 & 90 & 0.64 \\
Start from left hand - End with right hand & 2135 & 6482 & 0.2478 \\
Start from right hand - End with left hand & 1370 & 390 & 0.7784 \\
\hline
\end{tabular}

3) Crouched stance: In this case, a crouching posture of the robot is used for simulation. The mass of the object is set to $15 \mathrm{Kg}$ and the distance threshold between the CoM of the robot-object system and the support polygon is set to 60 $\mathrm{mm}$. The object CoM is located at $(-150,80,150) \mathrm{mm}$ in the object local coordinate system. The recorded data from the 

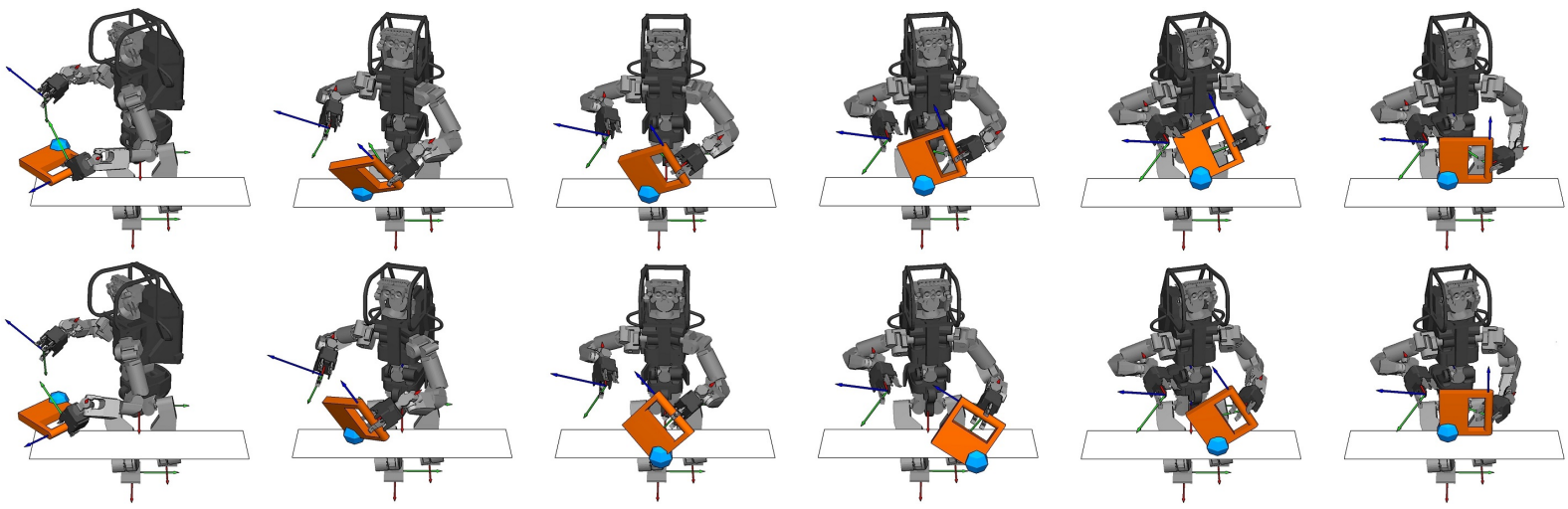

Fig. 1: Comparison between planned motion with different thresholds. In the upper case, the planner is given a $30 \mathrm{~mm}$ threshold whilst in the lower case the threshold is $0 \mathrm{~mm}$. The difference in constraints causes the planner to generate an alternative motion, preventing the object CoM, represented by the blue sphere, from traveling too far from the support polygon when the threshold is higher.
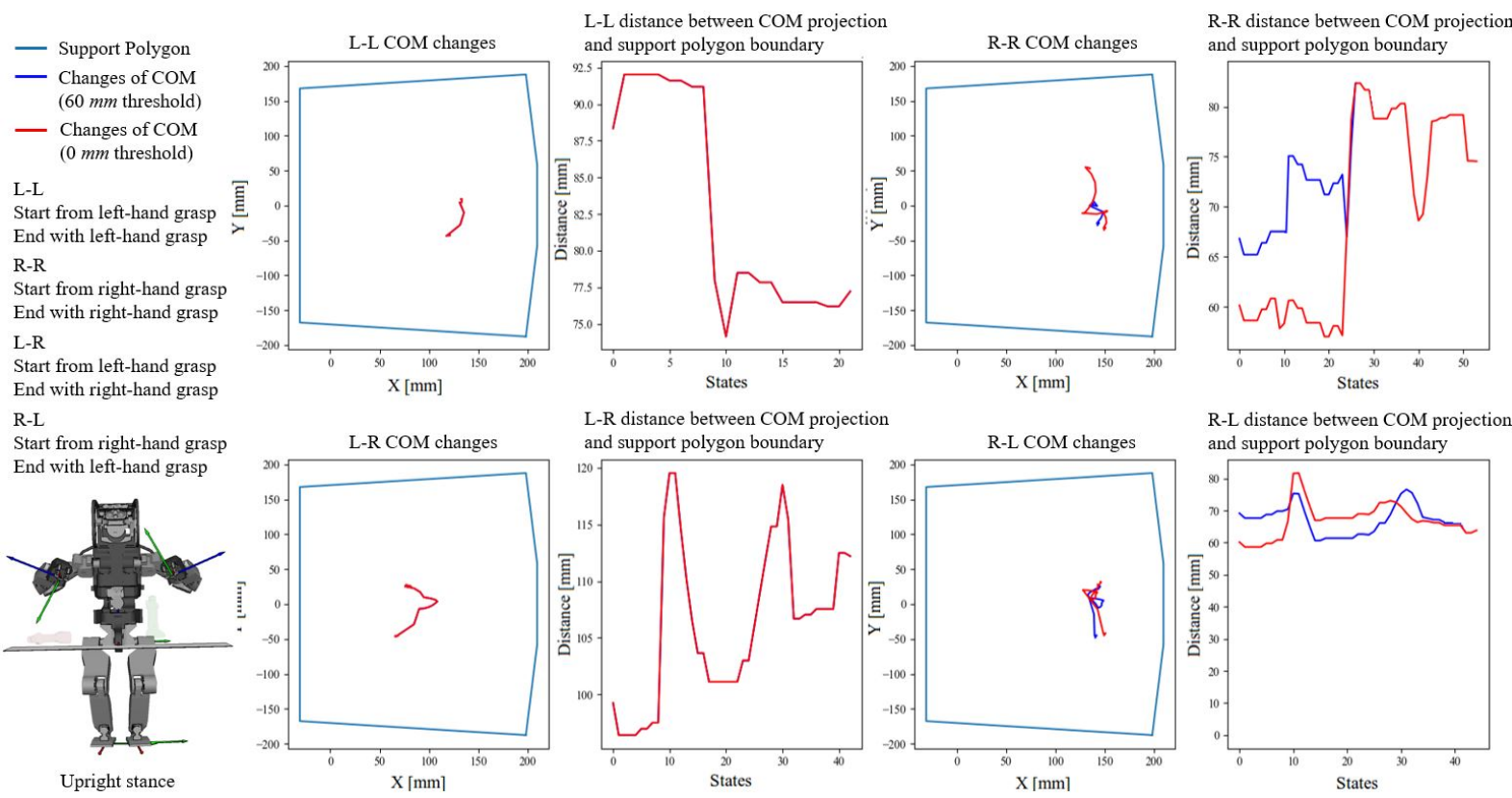

Fig. 2: CoM changes for the robot-object system while performing regrasp tasks with an upright stance and different hand configurations and thresholds. The results show the different CoM changes generated by the planner to maintain the stability of the task over two given thresholds. NOTE: When the results are the same for $0 \mathrm{~mm}$ and $60 \mathrm{~mm}$ thersholds, the blue curves $(60 \mathrm{~mm})$ are hidden by the red curves $(0 \mathrm{~mm})$. All plots in following figures are the same.

task stability measurement is shown in Table III Comparison of CoM changes with threshold $60 \mathrm{~mm}$ and $0 \mathrm{~mm}$ is shown in Fig 4

TABLE III: Crouched Stance: Path evaluations

\begin{tabular}{lccc}
\hline Hand configuration & $C_{\text {hand }}$ & $U_{\text {hand }}$ & $R_{\text {hand }}$ \\
\hline Start from left hand - End with left hand & 12242 & 0 & 1 \\
Start from right hand - End with right hand & 654 & 0 & 1 \\
Start from left hand - End with right hand & 6901 & 0 & 1 \\
Start from right hand - End with left hand & 14754 & 0 & 1 \\
\hline
\end{tabular}

4) One legged stance: For the final set of simulations, the robot was given a highly unstable posture, a one-legged stance with the right foot set directly under the robot CoM and the left foot raised $50 \mathrm{~mm}$ above the ground. The robot is required to perform a regrasp task with a bar-like object with $5 \mathrm{Kg}$ of mass. The object CoM is located at coordinates $(0,0,200) \mathrm{mm}$ and the stability threshold for the task is set to $30 \mathrm{~mm}$. The data gathered from this experiment is shown in table IV. The comparison with threshold $0 \mathrm{~mm}$ is shown in Fig 5

TABLE IV: One-Legged Stance: Path evaluations

\begin{tabular}{lccc}
\hline Hand configuration & $C_{\text {hand }}$ & $U_{\text {hand }}$ & $R_{\text {hand }}$ \\
\hline Start from left hand - End with left hand & 2628 & 0 & 1 \\
Start from right hand - End with right hand & 4299 & 2401 & 0.6416 \\
Start from left hand - End with right hand & 2558 & 2444 & 0.5113 \\
Start from right hand - End with left hand & 1306 & 88 & 0.9368 \\
\hline
\end{tabular}




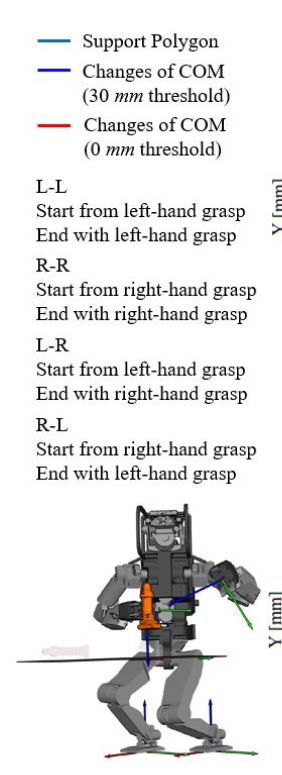

Staggered stance
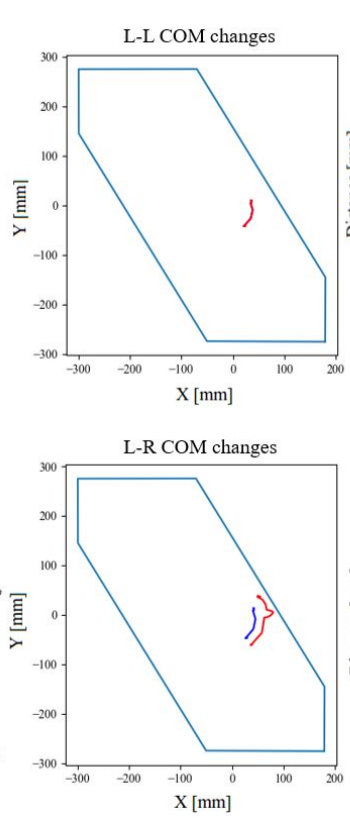

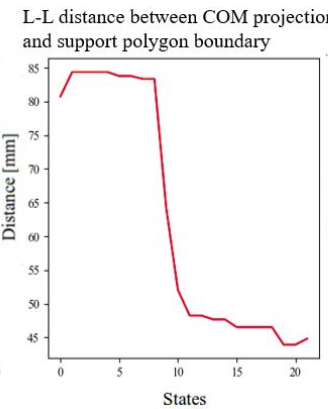

L-R distance between COM projection and support polygon boundary

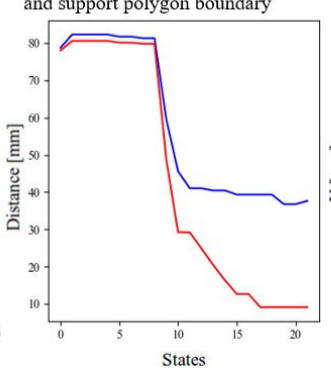

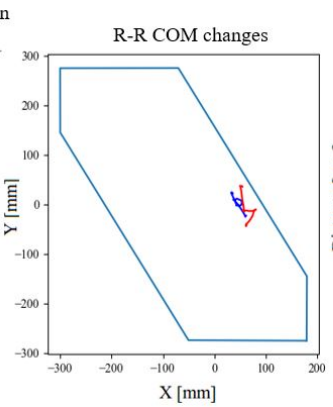

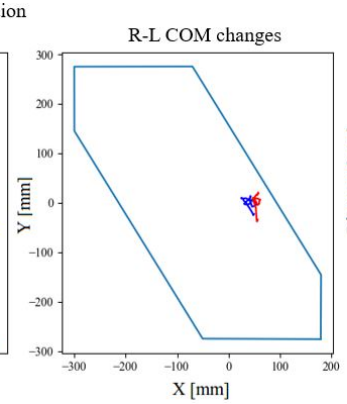

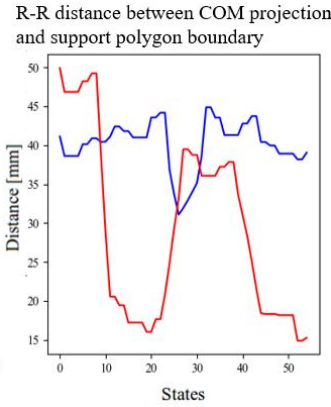

R-L distance between COM projection and support polygon boundary

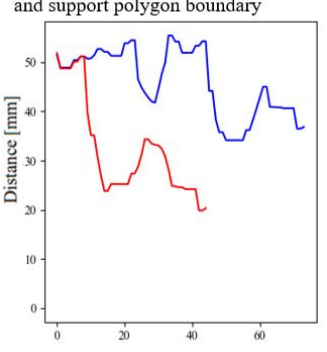

Fig. 3: CoM changes of the system while performing regrasp with a staggered stance and different hand configurations and thresholds.

\section{B. Analysis of the simulation results}

For the upright stance, the planner did not need to remove unstable poses from the regrasp graph when the robot used a left-handed one-armed configuration, or when it used a LeftRight dual-armed configuration, making the planned motions for the different thresholds equal as seen in Fig 2 On the other hand, some path corrections were performed by the planner for the right hand configuration and left-right hand configuration. The results show that the system CoM tends to move more towards the support polygon edge when the robot finishes its task using the right hand, but the planner is able to generate a path that keeps the CoM distance above the given threshold. These results also agree with the data shown in Table 1 which illustrates the left and left-right hand configurations as the most stable for the task.

For the staggered stance, the robot showed higher stability while performing the regrasp task using only its left arm, with 0 unstable poses recorded while the robot executed the task and a task evaluation of 0.9795 as seen in Table $\mathrm{I}$ and Fig 3 . Meanwhile, the most unstable hand configurations involve right hand being used to put the object in its final position, this shows that the twisting motion performed by the robot to lower down the object to its goal position with its right hand introduces undesired instability for this particular task. The staggered position changes the robot support polygon making the robot balance more susceptible to sideways motions. Since the right foot did not change positions, compared to the previous experiment, the stability of action of picking up the object, which is located on the right-hand side of the robot, does not change noticeably. On the other hand, since the left leg does not provide as good a support compared to the previous experiments, sideways motions to the left-hand side of the robot introduce instability to the robot.

The crouched stance aligns the robot CoM with its support polygon and also lowers its height. The posture puts the robot in a more stable state, which allowed us to increase the weight of the object to $8 \mathrm{Kg}$ and the threshold to 60 $\mathrm{mm}$ while still guaranteeing stable states for planning. With a stability ratio of 1 and 0 unstable poses encountered as seen in Table II and Fig 4, we were able to find a stance that maximizes the stability of the task, making the task stability measurement a useful tool for finding not only the most stable hand configuration, but also to compare the stability of different stances for the same task.

The staggered stance is challenging. The results indicate that the left-hand configuration is the most stable choice for the robot, with a stability ratio of 1 , as shown in table IV These results show that twisting motion performed by the use of the right hand to put the object in its goal pose introduces instability to the robot-object system, as seen in Fig 5 . Since the left leg is not supporting the body and it does not move with the rotation of the waist, there is more unsupported mass on the left-hand side. When the robot twists its waist to place the object with the right hand, the CoM shifts further to the left, making the task more unstable. Note that the constraints for the one-legged stance are softened. The stability ratio is therefore sometimes higher than other stances.

In several cases, the comparison between CoM changes with a threshold of $0 \mathrm{~mm}$ and other threshold values results in a noticeable difference in the robot motions, as seen in Fig 11 On the other hand, when the conditions of the robotobject system allow the robot to perform the task without incurring in unstable poses for a given threshold, the planner generates the same motion for lower threshold values, as seen in Fig 4 Since the planner does not necessarily generate the 

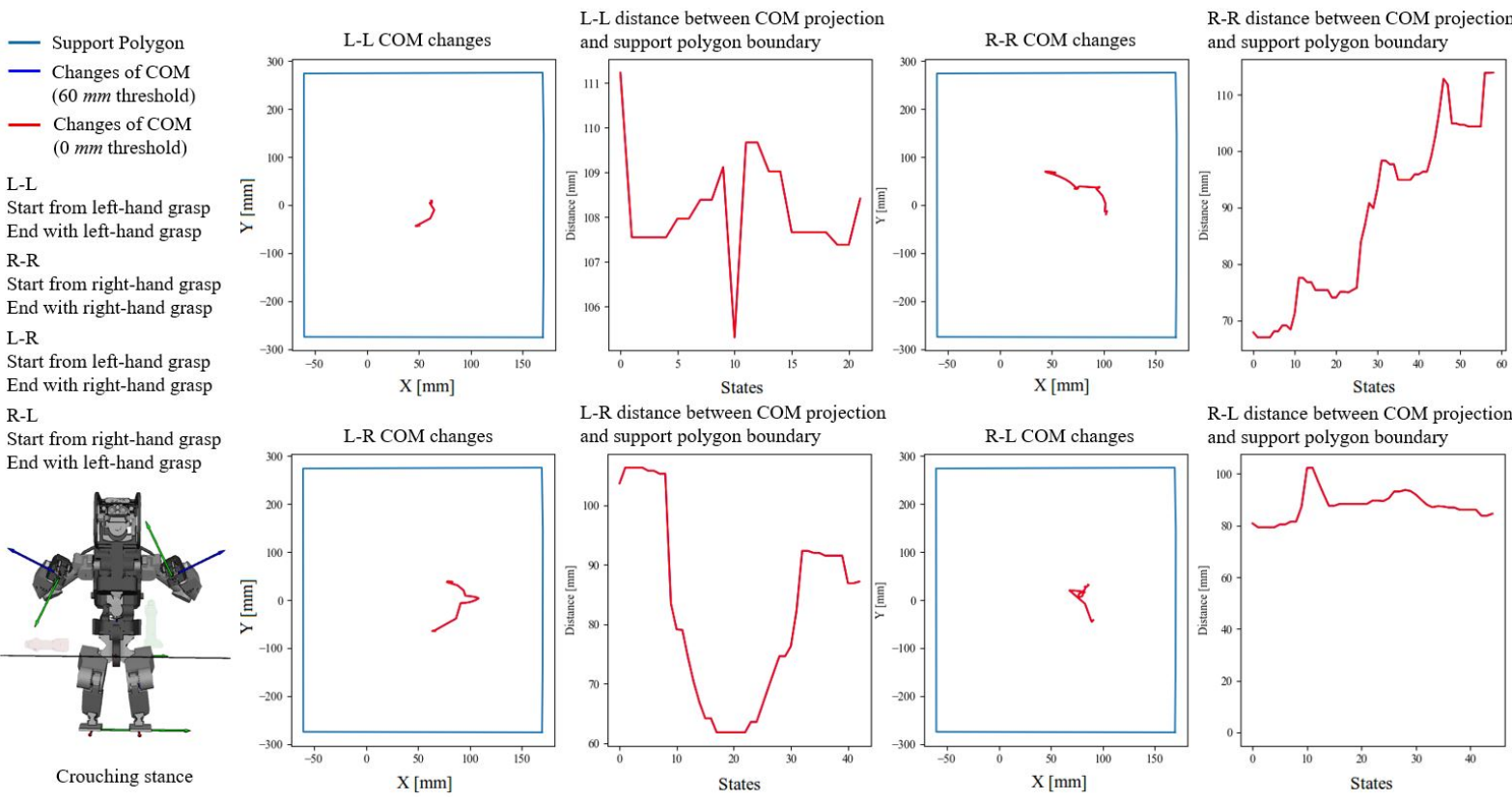

R-L distance between COM projection and support polygon boundary

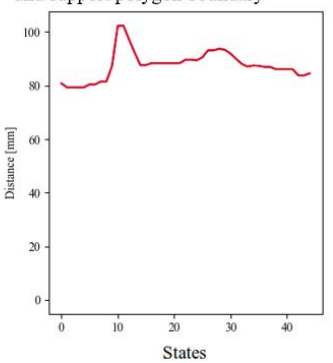

Fig. 4: CoM changes of the system while performing regrasp with a crouched stance and different hand configurations and thresholds.

most stable path but assures that it maintains a minimum of stability, these results are expected. Meanwhile, the planning time is still comparable to [2].

\section{Real-world experiments}

Following the results obtained in the simulations, we implemented our CoM-restricted regrasp planner in the HRP5P humanoid robot platform. The HRP5P represents the 5th generation of the Humanoid Robotics Project [3]. In this case, the planning of the robot motions was performed offline with our simulator. The motion sequences generated for this tasks were then given to the robot to perform. The setting and environment of the robot are composed by a table, an electric drill and a small box as seen in Fig 6(a).

To test the planner, the robot was given two dual-arm regrasping tasks. In the first task, the robot is required to move the drill by picking it up using a right-hand grasp and placing it down using a left-hand grasp. The planner is able to generate a motion sequence for the robot while keeping the robot-object system CoM above a minimum threshold distance to the support polygon edges of $55 \mathrm{~mm}$. The execution of the planned result is shown in Fig 6 a). In the second task, the object is laying sideways initially and the goal is to reorient it to an upright pose. The robot successfully completed the task without tipping over or colliding with its environment or itself. The execution is shown in Fig 6 (b). Details are in the supplementary video.

\section{Conclusions}

This paper presented a CoM based planner that planned regrasp motions while preserving robot balance. Simulation results showed that the planner eliminates several robot states that could be too unstable for the robot, generating different motions that keep the system CoM distance to the support polygon edges above the given threshold without noticeably increasing computation time. The results also revealed that the proposed task stability measurement could be used to evaluate the stability of a task with uncommon, or suboptimal stances which a robot might be forced to take, for reasons such as obstacles or damaged parts. The simulation results were executed by an HRP5P robot, demonstrating its practicality.

\section{REFERENCES}

[1] W. Wan and K. Harada, "Reorientating objects with a gripping hand and a table surface," in Humanoids, 2015, pp. 101-106.

[2] - "Developing and comparing single-arm and dual-arm regrasp," $R A L$, vol. 1, no. 1, pp. 243-250, 2016.

[3] H. Inoue, H. Hirukawa et al., "Hrp: Humanoid robotics project of miti," Journal of RSJ, pp. 2-7, 2001.

[4] S. M. LaValle and J. J. Kuffner Jr, "Randomized kinodynamic planning," IJRR, vol. 20, no. 5, pp. 378-400, 2001.

[5] S. A. Stoeter, S. Voss et al., "Planning of regrasp operations," in ICRA, vol. 1, 1999, pp. 245-250.

[6] T. Lozano-Pérez et al., Handey: a robot task planner. MIT Prs., 1992.

[7] T. Siméon et al., "Manipulation planning with probabilistic roadmaps," IJRR, vol. 23, no. 7-8, pp. 729-746, 2004.

[8] K. Kaneko et al., "Humanoid robot hrp-3," in IROS, 2008, pp. 2471-8.

[9] N. G. Tsagarakis et al., "Walk-man: A high-performance humanoid platform for realistic environments," JFR, vol. 34, no. 7, pp. 1225-59, 2017.

[10] O. Stasse et al., "Talos: A new humanoid research platform targeted for industrial applications," in Humanoids, 2017.

[11] L. Chen et al., "Manipulation planning to keep an object stable under a sequence of external forces," arXiv preprint arXiv:1710.11190, 2017.

[12] D. Kanoulas et al., "Center-of-mass-based grasp pose adaptation using 3d range and force/torque sensing," IJHR, p. 1850013, 2018.

[13] B. Sundaralingam and T. Hermans, "Geometric in-hand regrasp planning: Alternating optimization of finger gaits and in-grasp manipulation," arXiv preprint arXiv:1804.04292, 2018.

[14] D. Berenson et al., "Manipulation planning on constraint manifolds," in ICRA, 2009, pp. 625-632. 


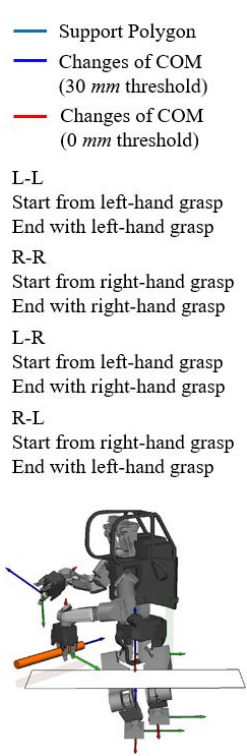

One-legged stance
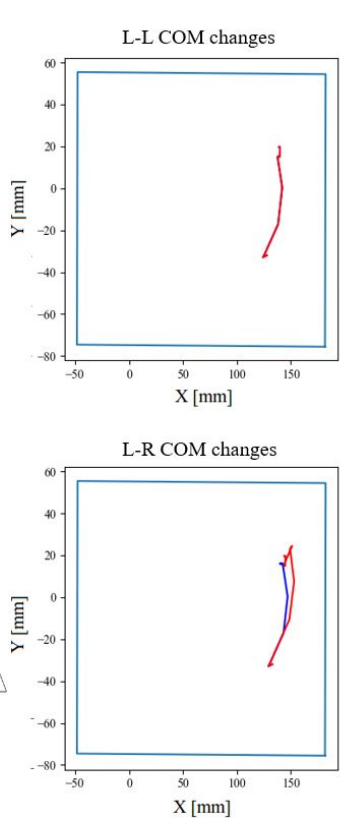

L-L distance between COM projection and support polygon boundary

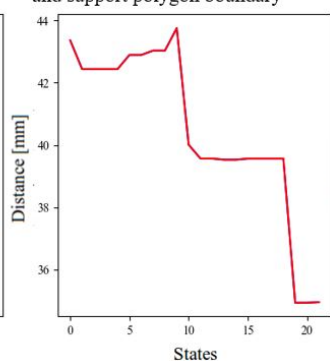

L-R distance between COM projectio and support polygon boundary

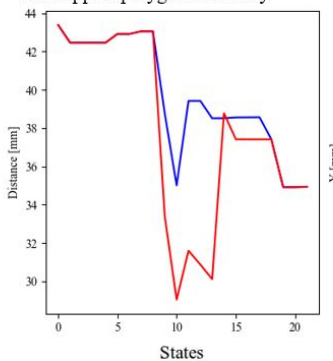

R-R COM changes

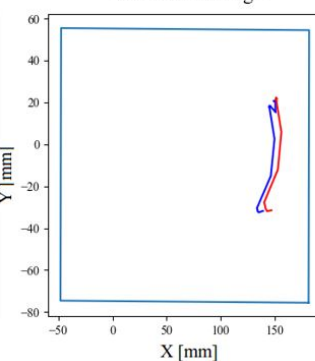

R-L COM changes

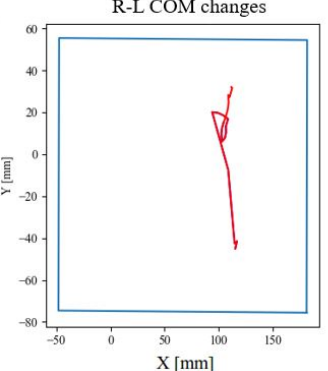

R-R distance between COM projection and support polygon boundary

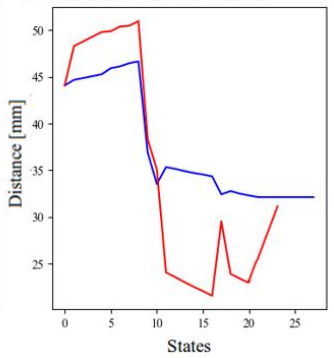

R-L distance between COM projection and support polygon boundary

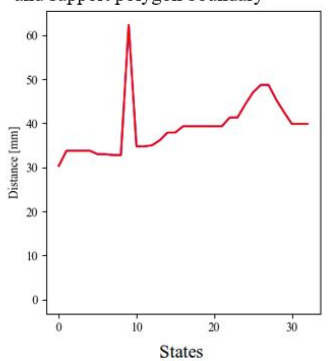

Fig. 5: CoM changes of the system while performing regrasp with a one-legged stance and different hand configurations and thresholds.

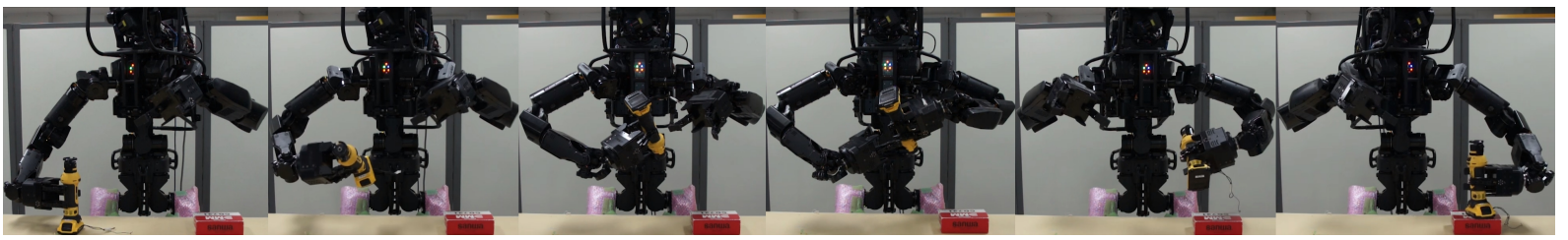

(a) The HRP5P performing a dual-arm regrasp task. The robot is commanded to move the drill using a right-to-left-hand regrasp.

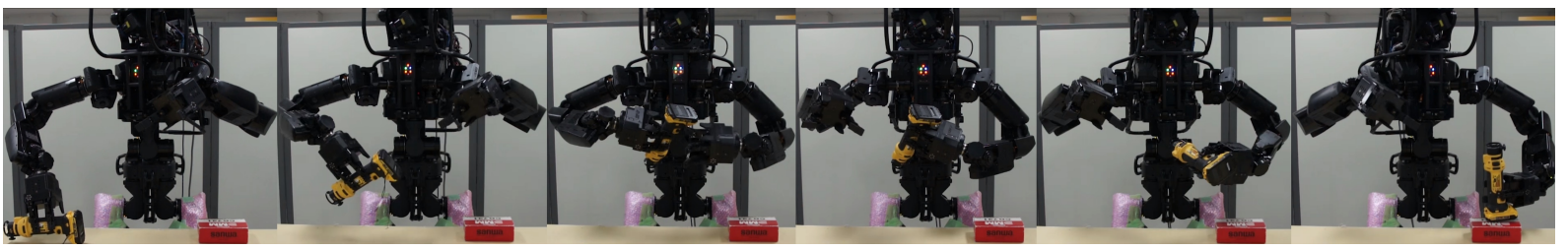

(b) The second dual-arm regrasp task. In this case, the object is laying sideways initially. The goal is in an upright pose.

Fig. 6: Motion sequences of the HRP5P robot reorienting an electric drill using dual-arm regrasp.

[15] J. Schulman et al., "Motion planning with sequential convex optimization and convex collision checking," IJRR, vol. 33, no. 9, pp. 12511270, 2014.

[16] E. Garcia et al., "A classification of stability margins for walking robots," Robotica, vol. 20, no. 6, pp. 595-606, 2002.

[17] D. E. Orin, "Interactive control of a six-legged vehicle with optimization of both stability and energy," Ph.D. dissertation, The Ohio State University, 1976.

[18] G. Nava et al., "Stability analysis and design of momentum-based controllers for humanoid robots," in IROS, 2016, pp. 680-687.

[19] C. Ott, B. Henze, G. Hettich, T. N. Seyde, M. A. Roa, V. Lippi, and T. Mergner, "Good posture, good balance: Comparison of bioinspired and model-based approaches for posture control of humanoid robots," $R A M$, vol. 23, no. 1, pp. 22-33, 2016.

[20] B. Henze, C. Ott, and M. A. Roa, "Posture and balance control for humanoid robots in multi-contact scenarios based on model predictive control," in IROS, 2014, pp. 3253-3258.

[21] T. Koolen, M. Posa, and R. Tedrake, "Balance control using center of mass height variation: limitations imposed by unilateral contact," in Humanoids, 2016, pp. 8-15.
[22] C. G. Atkeson et al., "No falls, no resets: Reliable humanoid behavior in the darpa robotics challenge," in Humanoids, 2015, pp. 623-630.

[23] M. Brunner et al., "Design and comparative evaluation of an iterative contact point estimation method for static stability estimation of mobile actively reconfigurable robots," RAS, vol. 63, pp. 89-107, 2015.

[24] K. Harada et al., "A humanoid robot carrying a heavy object," in Humanoids, 2005, pp. 1712-1717.

[25] M. Stilman, J. Olson, and W. Gloss, "Golem krang: Dynamically stable humanoid robot for mobile manipulation," in ICRA, 2010, pp. 3304 3309.

[26] K. H. Teja, F. James, and S. V. Shah, "Optimal whole body motion planning of humanoid with articulated spine for object manipulation in double support phase," in ICAR, 2015, p. 30.

[27] I. G. Ramirez-Alpizar et al., "Motion generation for pulling a fire hose by a humanoid robot," in Humanoids, 2016, pp. 1016-1021.

[28] R. B. McGhee and A. A. Frank, "On the stability properties of quadruped creeping gaits," Mathematical Biosciences, vol. 3, pp. 331$351,1968$.

[29] L. Jaillet, J. Cortés, and T. Siméon, "Transition-based rrt for path planning in continuous cost spaces," in IROS, 2008, pp. 2145-2150. 